\title{
Inhibition of Aspergillus fumigatus Biofilm and Cytotoxicity Study of Natural Compound Cis-9- Hexadecenal
}

\section{Shanu Hoda (D), Lovely Gupta, Harshita Agarwal, Gaurav Raj, Maansi Vermani and Pooja Vijayaraghavan*}

Antimycotic and Drug Susceptibility Laboratory, J-3 Block, Amity Institute of Biotechnology, Sector-125, Amity University, Uttar Pradesh, Noida, India.

\begin{abstract}
Clinical limitations and drug-resistance are the basis of the search for new antifungal therapeutics against biofilm forming pathogen Aspergillus fumigatus. Cis-9-hexadecenal is a natural compound present in various plants extracts, but its antifungal activity against $A$. fumigatus is still unexplored. The aim of present study was to evaluate the antifungal efficacy of cis-9-hexadecenal on $A$. fumigatus, specifically its biofilm and to assess its cytotoxicity. Broth micro-dilution method revealed that cis-9hexadecenal inhibited $90 \%$ of planktonic growth of $A$. fumigatus at $0.078 \mathrm{mg} / \mathrm{ml}$. In-vitro combinatorial effect between drug amphotericin B and cis-9-hexadecenal was studied by checkerboard assay. Cis9-hexadecenal, in combination to amphotericin-B had additive effect and showed enhanced drug efficacy against $A$. fumigatus. The effect of cis-9-hexadecenal on pre-formed biofilm was analyzed through 3-(4,5-dimethylthiazol-2-yl)-2,5-diphenyltetrazolium bromide (MTT) assay and scanning electron microscopy. In MTT assay, minimum biofilm-eradicating concentration-80 of cis-9-hexadecenal was $0.156 \mathrm{mg} / \mathrm{ml}$. Scanning electron micrograph showed absence of extracellular matrix and tangled hyphae in cis-9-hexadecenal treated biofilm whereas these structures were present in both untreated and amphotericin-B treated biofilm. Drug-likeness was predicted through in-silico ADMET studies. The study envisaged that cis-9-hexadecenal followed Lipinski's rule but had a higher Log P value indicating that it's not hydrophilic. The compound can be modified to enhance the absorption and permeation. Cell cytotoxicity study exhibited that cis-9-hexadecenal was not toxic to normal human lung epithelial cell line L-132 upto $0.62 \mathrm{mg} / \mathrm{ml}$. The study concluded that cis-9-hexadecenal might be explored further as a potential therapeutic molecule for $A$. fumigatus associated diseases.
\end{abstract}

Keywords: Biofilm, Aspergillus fumigatus, natural compound, cis-9-hexadecenal, cytotoxicity.

*Correspondence: vrpooja@amity.edu; +91-9650548930

(Received: 12 March 2019; accepted: 27 April 2019)

Citation: Shanu Hoda, Lovely Gupta, Harshita Agarwal, Gaurav Raj, Maansi Vermani and Pooja Vijayaraghavan, Inhibition of Aspergillus fumigatus Biofilm and Cytotoxicity Study of Natural Compound Cis-9-hexadecenal, J Pure Appl Microbiol., 2019; 13(2): 1207-1216. doi: 10.22207/JPAM.13.2.61

C The Author(s) 2019. Open Access. This article is distributed under the terms of the Creative Commons Attribution 4.0 International License which permits unrestricted use, sharing, distribution, and reproduction in any medium, provided you give appropriate credit to the original author(s) and the source, provide a link to the Creative Commons license, and indicate if changes were made. 


\section{INTRODUCTION}

Fungal biofilm is a complex form of fungal growth embedded in extracellular matrix (ECM) which irreversibly adheres to any superficial layers like cell surfaces and various medical devices (Nett and Andes, 2015). The ECM is composed of polysaccharides and facilitates attachment to the substrates in vitro and in vivo. It binds and stabilises fungal colonies to form complex structure with each other, which can be easily detected through chemical assays and by microscopic studies (Lagree et al., 2018). The biofilms are more challenging to human health because their structural complexity lowers fungal growth rate and decrease the antifungal drug susceptibility. Thus, fungal biofilms are difficult to eradicate (Sav et al., 2018).

Various fungal species have been reported to form biofilm including Aspergillus, Candida, Cryptococcus and Fusarium (Sav et al., 2018; Martinez and Casadevall, 2007; Fanning and Mitchell, 2012). Aspergillus fumigatus is a ubiquitous opportunistic fungal pathogen that accounts for various fatal lung diseases like invasive pulmonary aspergillosis, allergic bronchopulmonary aspergillosis and aspergilloma in immuno compromised hyper sensitive individuals. In lungs, $A$. fumigatus has been reported to form a hydrophobic biofilm comprised of numerous intertwined hyphae covered with ECM (Tseung and Zhao, 2016). This complex structure resists the therapeutics up to 1000 -folds in comparison to free colonies (Liu et al., 2012). The efficacy of existing antifungals in eradicating biofilms is limited and is always questionable in context to their safety and efficacy. The treatment of biofilmrelated Aspergillus infection is becoming a serious clinical challenge, emphasising the need for new therapeutic agents. One prospect to control biofilm formation is to develop new drugs using natural compounds that are commonly known to be safe (Boominathan and Ramamurthy, 2009).

Natural extracts and their derived compounds play an important role in modern drug development due to their easy availability and minimal side effects (Veeresham, 2012). Numerous plant extracts have been reported to possess antifungal activities. Cis-9-hexadecenal (C-9-H) is a bioactive natural compound, commonly known as palmitolealdehyde and is reported to be present in various plant extracts including Myristica fragrans,
Thuja orientalis, Pentaclethramacrophylla Benth and Cuminum cyminum (Ololade et al., 2014; Fang et al., 2018; Olasehinde et al., 2018). It belongs to group of organic $C_{16}$ mono-unsaturated fatty aldehydes which has been reported to possess antimicrobial and anti-inflammatory effect (Mujeeb et al., 2014; Kumar and Rajakumar, 2016; Juliet et al., 2018). It has been described as aliphatic long chain volatile compound that helps in fatty acid degradation and has characteristic strong fragrance (Huang et al., 2016). It has also been used to synthesise lipidised-soluble and long-acting insulin to enhance hypoglycaemic effect (Mei et al., 1999). However, its antifungal activity against $A$. fumigatus and its biofilm is still unexplored, so this component can be explored more for medicinal prospect. There are limited studies on the effect of natural compounds on $A$. fumigatus biofilms (Liu et al., 2011). Therefore, the objective of the present study was to investigate the antifungal efficacy of a natural compound cis-9hexadecenal on A. fumigatus biofilm and to assess its cytotoxicity. Combining conventional drugs and traditional medicine may represent a useful approach to combating antifungal infections (Yang et al., 2018). Therefore, the combined antifungal activity of $\mathrm{C}-9-\mathrm{H}$ with amphotericin $\mathrm{B}(\mathrm{AmpB})$ against $A$. fumigatus colonies was investigated via a broth micro-dilution checkerboard assay. Biofilm eradicating potential of the natural compound C-9-H was explored using 3-(4,5-dimethylthiazol2-yl)-2,5-diphenyltetrazolium bromide (MTT) assay (quantitative approach) and scanning electron microscopy (SEM; analytical approach). Besides, the cytotoxicity assay of the compound was also conducted both on cell lines and through in-silico approach.

\section{MATERIALS AND METHODS Collection of $A$. fumigatus fungal strain}

A. fumigatus strain (ATCC-46645) was a kind gift from Prof. Axel Brakhage, Department of Molecular and Applied Microbiology, Lelbeniz Institute for Natural Product Research and Infection Biology- HKI, Germany. The strain was cultured on Czapek-Dox Agar (CzA) at $28 \pm 2^{\circ} \mathrm{C}$ for five days to obtain conidial growth. Culture was maintained monthly by sub-culturing on $\mathrm{CzA}$ slants. 
Procurement of natural compound C-9-H and its identification using gas chromatography-mass spectroscopy

The natural compound $\mathrm{C}-9-\mathrm{H}\left(\mathrm{C}_{16} \mathrm{H}_{30} \mathrm{O}\right)$ was procured from Carbosynth (Funakoshi Co, Japan) and characterized by Gas Chromatography coupled with Mass Spectroscopy (GCMS) at Advanced Instrumentation and research Facility, Jawaharlal Nehru University, Delhi (India). The instrument GCMS-QP2010 Plus (Shimadzu, Kyoto, Japan) with Rtx-5 MS capillary column (30 m length $x 0.25 \mathrm{~mm}$ diameter $\times 0.25 \mu \mathrm{m}$ film thickness) was used for GCMS analyses. It was equipped with an auto injector, head space sampler, a mass selective detector with an ion source $\left(220^{\circ} \mathrm{C}\right)$ and an interface $\left(260^{\circ} \mathrm{C}\right)$. The injector was set at $250^{\circ} \mathrm{C}$ with a split ratio of 1:50. MS was taken at threshold of $1000 \mathrm{eV}$ with a mass range of $\mathrm{m} / \mathrm{z} 40-650$. The oven temperature was programmed from $60^{\circ} \mathrm{C}(3$ min initially) to $280^{\circ} \mathrm{C}$ at $10^{\circ} \mathrm{C} / \mathrm{min}$. Helium was used as a carrier gas at $1.21 \mathrm{ml} / \mathrm{min}$ of column flow according to stranded methods (Das et al., 2014). The compound was identified on the basis of retention time (RT) by gas chromatography and interpretation of mass spectrum was performed by comparing spectral fragmentation obtained which was matched to the database provided by National Institute of Standards and Technology (NIST) library and Wiley8 library (Das et al., 2014; Adams, 2007).

The compound was solubilised in $100 \%$ dimethyl sulfoxide (DMSO) to make $100 \mathrm{mg}$ / $\mathrm{ml}$ final concentration. Before performing any experiments, stock solutions in DMSO were further diluted in Czapek-Dox Broth (CzB) so that the final concentration of DMSO did not exceed an amount that had any detectable effect in assays (Szumilak et al., 2017).

Determination of Minimum inhibitory concentration (MIC) for A. fumigatus

The MIC values were determined by standard Clinical and Laboratory Standards Institute (CLSI) broth micro-dilution method in sterile 96-well flat bottom micro-plates with minor modifications (CLSI, 2008). Two-fold serial dilutions were made in $\mathrm{CzB}$ to give final concentrations of $5-0.009 \mathrm{mg} / \mathrm{ml}$ for $\mathrm{C}-9-\mathrm{H}$, and $50-0.09 \mu \mathrm{g} / \mathrm{ml}$ for amphotericin-B (AmpB). One hundred microlitre of $A$. fumigatus conidial suspension $(0.8 \mathrm{x}$ $10^{4}$ conidia/mL) was added to each well. Negative control comprised $\mathrm{CzB}$ only while the positive control was $\mathrm{CzB}$ with conidial suspension. The final volume of each well was $200 \mu$ l. The MICs of C-9-H and AmpB were observed after 48-96 $\mathrm{h}$ incubation at $28 \pm 2^{\circ} \mathrm{C}$. MIC was defined as the lowest concentration of the compound/drug that exhibited complete inhibition of microbial growth.

\section{In-vitro combinatorial evaluation}

The combined effect of the compound $\mathrm{C}-9-\mathrm{H}$ with the antifungal drug AmpB was evaluated by checkerboard micro-dilution method and the type of interaction was predicted on the basis of fractional inhibitory concentration index (FICl; Fratini et al., 2017). Eight concentrations of C-9-H and AmpB were prepared (16 MIC, 8 MIC, $4 \mathrm{MIC}, 2 \mathrm{MIC}, \mathrm{MIC}, \mathrm{MIC} / 2, \mathrm{MIC} / 4$ and $\mathrm{MIC} / 8$ ). The compound was diluted two-fold in vertical orientation, while the AmpB was diluted two-fold in horizontal orientation in the 96-well micro-titer plate using $\mathrm{CzB}$. The final volume of each well was $100 \mu \mathrm{l}$ comprising 1:1 ratio of each tested sample dilution. Lastly, $100 \mu$ l of $A$. fumigatus suspension ( $1 \times 10^{5}$ conidia/ml) was added to each well in order to obtain the FIC final concentrations $8 \mathrm{MIC}$, $4 \mathrm{MIC}, 2 \mathrm{MIC}, \mathrm{MIC}, \mathrm{MIC} / 2, \mathrm{MIC} / 4, \mathrm{MIC} /$ and $\mathrm{MIC} / 16$ as illustrated in Fig. 1. $\mathrm{CzB}$ was set as negative control whereas, positive control was $\mathrm{CzB}$ with $A$. fumigatus suspension. After $96 \mathrm{~h}$ incubation at 28 $\pm 2^{\circ} \mathrm{C}$, the $\mathrm{FICl}$ was calculated as follows: $\mathrm{FICl}=\mathrm{FIC}$ $A+F I C B$, where, FIC A is the MIC of C-9-H in the combination/MIC of C-9-H alone, and FIC B is the MIC of AmpB in the combination/MIC of AmpB alone. The results were interpreted as synergy $(\mathrm{FICl} \leq 0.5)$, addition $(0.5<\mathrm{FICl} \leq 1)$, indifference $(1<\mathrm{FICl} \leq 2)$ or antagonism ( $\mathrm{FICl}>2$; Fratini et al., 2017).

Effect of C-9-H on A. fumigatus pre-formed Biofilm

The minimal biofilm-eradicating concentration $80\left(\mathrm{MBEC}_{80}\right)$ of the natural compound $\mathrm{C}-9-\mathrm{H}$ on pre-formed biofilm was calculated by MTT assay on a 96-well flat bottom microtiter plates with minor modifications (Sav et al., 2018). Briefly, the conidial suspension of A. fumigatus was prepared in phosphate buffer saline (PBS) supplemented with $0.025 \%$ Tween-20 and adjusted to $1 \times 10^{5}$ conidia/mL using CzB. The conidial suspension $(100 \mu \mathrm{L})$ was added to each well and incubated at $37^{\circ} \mathrm{C}$ for $24 \mathrm{~h}$ without agitation for biofilm formation. Then non-adherent 
conidia were removed by washing with PBS. Various concentrations of $\mathrm{C}-9-\mathrm{H}$ and antifungal drug amp-B were added to the respective wells and further incubated at $37^{\circ} \mathrm{C}$ statically for an additional 24h. Negative control wells contained only CzB media. Wells with preformed biofilms without any treatment were considered as positive control.

\section{Quantitative Analysis - MTT Reduction Assay}

The effect of $\mathrm{C}-9-\mathrm{H}$ and $\mathrm{AmpB}$ in the pre-formed biofilms was estimated using a semiquantitative viability based MTT reduction assay as per the protocol of Manavathu et al., 2014. The supernatants from the wells containing preformed biofilm were aspirated and the wells were washed twice with PBS. MTT solution $(5 \mathrm{mg} / \mathrm{ml})$ was prepared and filter-sterilized $(0.22 \mathrm{~mm}$ pore size filter). Freshly prepared menadione solution (0.2mM, 20:1 v/v) was immediately added to MTT-solution prior to the assay. Thereafter, MTTmenadione solution was transferred to each well and incubated in the dark for $3 \mathrm{~h}$ at $37^{\circ} \mathrm{C}$. MTT was removed from wells and washed thrice with distilled water. Ethanol (70\%) containing $0.1 \mathrm{~N} \mathrm{HCl}$ was added to dissolve the insoluble tetrazolium salt and incubated for another 30minutes at $37^{\circ} \mathrm{C}$. The optical density of the supernatant was measured at $570 \mathrm{~nm}$ with a micro-titre plate reader (Cloud-Clone smart microplate reader, Model no. SMR-16.1).

\section{Qualitative analysis- Scanning electron microscopy}

The $A$. fumigatus biofilms were cultured in a 12-well polystyrene plate at $\mathrm{MBEC}_{80}$ of $\mathrm{C}-9-\mathrm{H}$ and AmpB (Manavathu et al., 2014). For SEM analysis, the samples were processed as described by Gonzalez-Ramirez et al., 2016, with minor modifications. Briefly, the biofilms were washed with PBS and fixed with $2 \%$ glutaraldehyde for 2 $\mathrm{h}$. The base of the 12-well plates was cut using a hot blade to obtain the intact biofilm. Further, the biofilms were dehydrated by gradually increasing the ethanol concentration from $10 \%$ to $90 \%$ for $10 \mathrm{~min}$ and finally with absolute alcohol for an additional 20min. The biofilms were dried, mounted on aluminium sheet and coated with gold-palladium alloy. The $A$. fumigatus biofilm topology of treated and untreated biofilm was viewed under SEM (Zeiss SEM, MA EVO -18 Special Edition).
In-silico screening of compound for therapeutic activity

In-silico study was conducted to determine the $\mathrm{C}-9-\mathrm{H}$ drug-likeness and health effect prediction profile. The compound structure was drawn manually by ChemSketch (Advanced Chemistry Development, inc., ACD/Labs, freeware 12.0, Toronto, Canada). Molecular property and drug likeness was analysed using an online softwares ACD/I-Lab version 4.5 (Tambunan et al., 2017) and MolSoft LLC (3.5-0, San Diego, CA; Lalitha and Sivakamasundari, 2010) respectively. The parameters deployed to envisage the physicochemical properties of the compound and to describe its disposition within an organism are summarized in Table 1 (Lipinski et al., 2001; Yusof et al., 2014). Set of properties (1-5) mentioned in Table 1 is known as 'Lipinski's rule', which identifies compounds with good oral absorption (Lipinski et al., 2001).

Table 1. In-silico prediction of physico-chemical and pharmacokinetic property of cis-9-hexadecenal

\begin{tabular}{llll}
\hline S.N. & Property & Criterion & $\begin{array}{l}\text { In-silico } \\
\text { analysis }\end{array}$ \\
\hline 1 & Molecular weight & $<500$ & 238.41 \\
2 & Hydrogen donor & $<6.0$ & 0 \\
3 & Hydrogen acceptor & $<5.0$ & 1 \\
4 & Rotational bonds & $<10.0$ & 13 \\
5 & Polar Surface Area & $<150$ & 17.07 \\
6 & Log P (lipophilicity) & $<5$ & 6.29 \\
7 & Log S (Solubility) & - & -5.73 \\
8 & Permeability & $1 *$ & $0.08^{*}$ \\
& Jejenum & $10^{-6} \mathrm{~cm} / \mathrm{s}$ & $10^{-6} \mathrm{~cm} / \mathrm{s}$ \\
9 & Oral bioavailability & - & $30-70 \%$ \\
10 & Active transport & - & No \\
11 & Passive transport & - & Yes \\
12 & Drug likeness score & - & -1.27 \\
\hline
\end{tabular}

\section{Cell line culture conditions}

The lung epithelial normal cell line L-132 was procured from National Centre for Cell Science, Pune, India. They were cultured in Dulbecco Modified Eagle Medium supplemented with $10 \%$ foetal bovine serum, $1 \%$ penicillin/ streptomycin and $1 \% \mathrm{~L}$-glutamine. The cells were incubated at $37^{\circ} \mathrm{C}$ in a humidified atmosphere of $5 \% \mathrm{CO}_{2}$ and $95 \%$ air. The existing medium was replaced with fresh one every $24 \mathrm{~h}$. 


\section{Cytotoxicity assay of C-9-H and AmpB}

In-vitro cytotoxic activity of C-9-H was evaluated at various concentrations $(0.625-0.039$ $\mathrm{mg} / \mathrm{ml}$ ) against lung epithelial normal cell line $\mathrm{L}-132$ and was compared to that of AmpB using the MTT assay. The cells were harvested using trypsin EDTA (EDTA $0.25 \%+$ Trypsin $0.53 \mathrm{mM}$ ) and seeded into 96-well plate at a density of 1 $\times 10^{5}$ cells per well and cultured overnight. After $24 \mathrm{~h}$, plain media was decanted and cells were incubated with various dilutions of $\mathrm{C}-9-\mathrm{H}$ and the drug AmpB for 24h. The wells containing only media and cells were regarded as positive control and the wells possessing only media were considered as negative control. After that the media was removed from wells. Cells were washed twice with PBS and taken for MTT assay. MTT reagent $(5 \mathrm{mg} / \mathrm{ml}$ in PBS) was added to each well and incubated for $4 \mathrm{~h}$ at $37^{\circ} \mathrm{C}$. Further, MTT was removed and wells were washed with PBS. The resultant formazan was dissolved in DMSO and analysed at $570 \mathrm{~nm}$ using micro-plate reader. Each experiment was studied in triplicate. The relative cell viability in percentage was calculated as $\left(A_{570}\right.$ of treated samples/ $A_{570}$ of untreated samples)* 100 (Venkatraman et al., 2005; Jadeja et al., 2017).

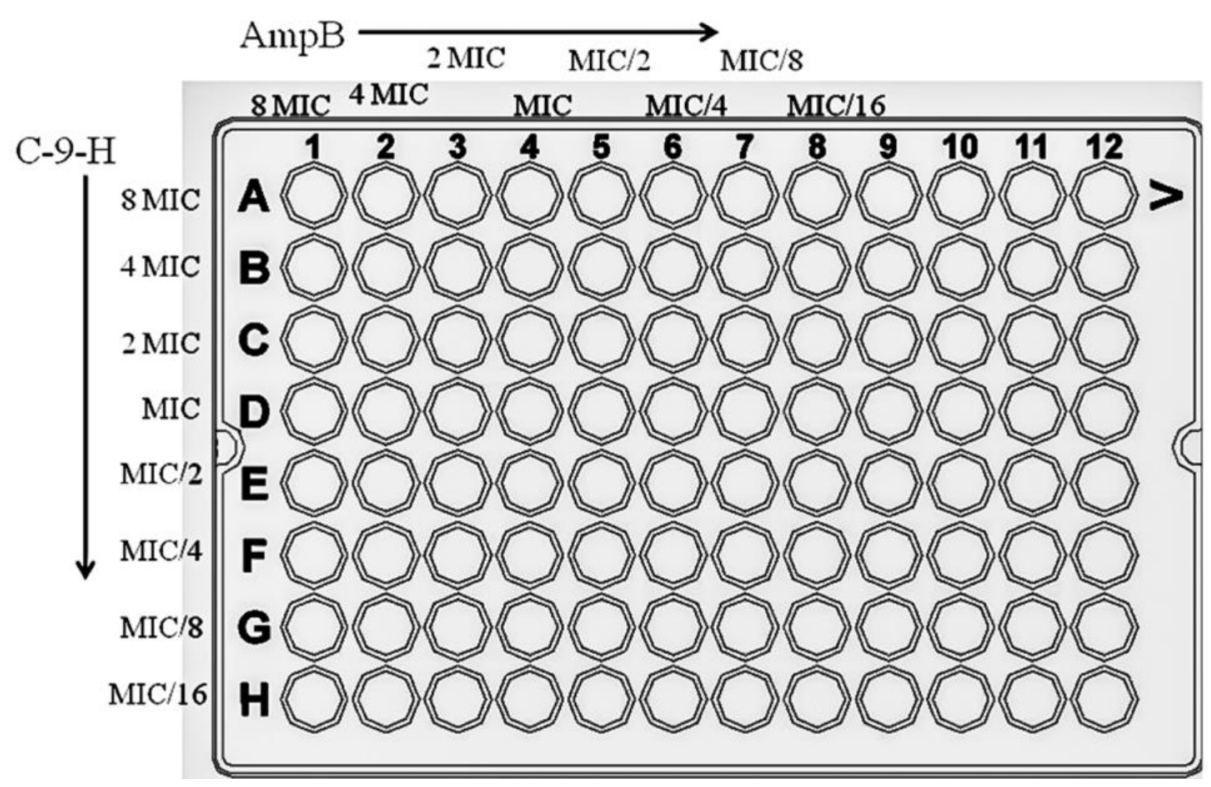

Fig. 1. Microdilution checkerboard method for FIC determination. Dilution of the drug amphotericin B was made horizontally whereas; cis-9-hexadecenal was diluted vertically. In both cases, the range of test sample was from $8 \mathrm{MIC}$ to $\mathrm{MIC} / 16$.

\section{RESULTS AND DISCUSSION \\ Identification of C-9-H using GCMS}

C-9-H was identified according to its mass spectra and relative retention indices determined on a non-polar stationary phase capillary column. GCMS analysis revealed the procured compound as C-9-H having peak area of $97.8 \%$ (Fig. 2). Table 2 depicts the chromatographic profile of the bioactive compound.

\section{Determination of minimum inhibitory concentration}

The limited spectrum of antifungal drugs against $A$. fumigatus, their serious side- effects, emergence of resistance and inefficacy against biofilms has created immense need for the development of new molecules exhibiting antifungal activity. C-9-H is a naturally occurring long chain unsaturated aldehyde, which has been

Table 2. Chromatographic profile of cis-9-hexadecenal

\begin{tabular}{lccc}
\hline Peak & Name & $\begin{array}{c}\text { Retention } \\
\text { time }\end{array}$ & Area \% \\
\hline 1 & 13-tetradecenal & 26.75 & 2.19 \\
2 & Cis-9-hexadecenal & 29.73 & 97.81
\end{tabular}




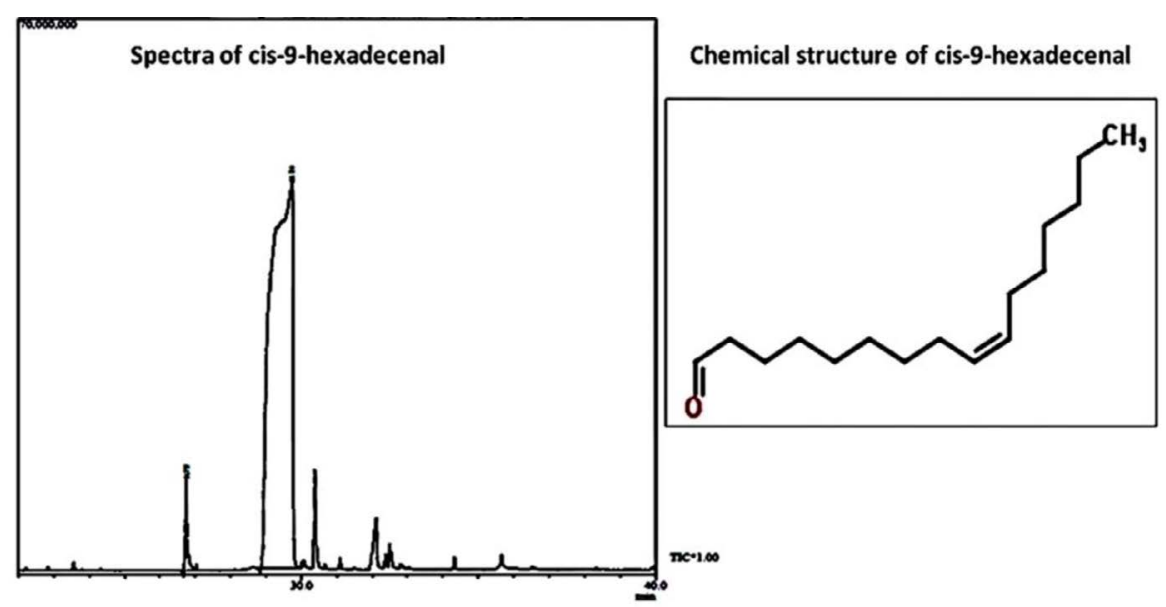

Fig. 2. GCMS chromatogram and chemical structure of the natural compound cis-9-hexadecenal.

reported to possess high value in pharmaceutical industries (Andewole et al., 2013). It has been reported that unsaturated aldehydes are highly reactive substances that readily react with the nucleophilic groups of the cell membrane, resulting in alteration of membrane permeability and leakage of intracellular materials. They exhibit a broad antimicrobial spectrum (Kubo et al., 1995; Bisignano et al., 2001; Trombetta et al., 2002).

In the present study, the antifungal activity of $\mathrm{C}-9-\mathrm{H}$ and $\mathrm{AmpB}$ against $\mathrm{A}$. fumigatus was assessed using the broth microdilution method. The MIC value of the $\mathrm{C}-9-\mathrm{H}$ was $0.078 \mathrm{mg} /$ $\mathrm{ml}$, while the MIC value for AmpB was $6.2 \mu \mathrm{g} / \mathrm{ml}$ (Table 3). According to CLSI document M38-A2, the $\mathrm{MIC}$ of AmpB against $A$. fumigatus ranges from $0.5-8 \mu \mathrm{g} / \mathrm{ml}$ (CLSI, 2008).

\section{In-vitro combinatorial evaluation}

The accurate prediction of synergy between a commercially available antifungal drug and a natural compound, based upon the results of in-vitro testing, is crucial for the determination of optimal doses of agents used in the combination antimicrobial therapy (Yang et al., 2018). In this study, the combinatorial effect of the compound C-9-H and drug AmpB was determined by the checkerboard method. The results of the checkerboard tests revealed decrease in the MIC with C-9-H and antifungal drug AmpB combination. Thus, $\mathrm{C}-9-\mathrm{H}$ enhances the therapeutic activity of AmpB against $A$. fumigatus due to additive interaction. The MIC for $A m p B$ was reduced almost 4 times, when used in combination with $\mathrm{C}-9-\mathrm{H}$.
According to the calculated $\mathrm{FICl}$ values (Table 3), the interactions of $\mathrm{C}-9-\mathrm{H}$ and $\mathrm{AmpB}$ was classified as additive $(0.5<\mathrm{FICl} \leq 1.0$; Yang et al., 2018).

Effect of C-9-H on A. fumigatus pre-formed Biofilm MTT assay for evaluation of biofilm formation

Biofilms are formed in most of the severe $A$. fumigatus infections which enhances persistence and antifungal tolerance; leading to significant morbidity and mortality. Combating biofilm would directly enhance the survival and life expectancy of infected individuals. Therefore, C-9-H was evaluated for its biofilm inhibiting activity.

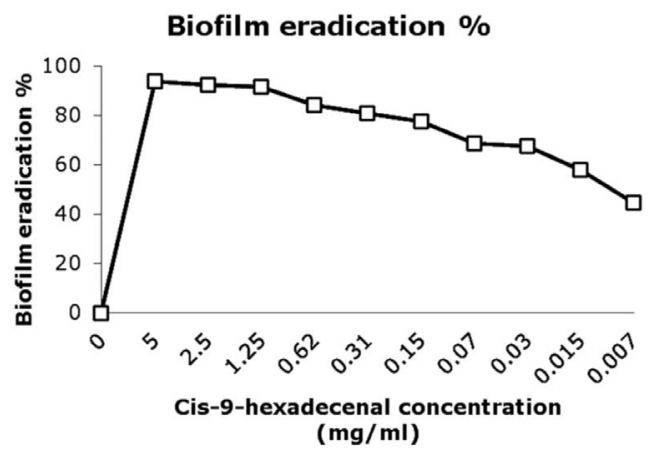

Fig. 3. Biofilm eradication percentage after treating the pre-formed biofilm with compound at various concentrations. The developed biofilms were treated with different concentrations of cis-9-hexedecenal for $24 \mathrm{~h}$ or $1 \%$ DMSO as positive control. MTT assay was done to determine the percentage eradication of biofilm formed. 
Table 3. Minimum inhibitory concentration (MIC) and fractional inhibitory index (FICI) of cis-9-hexadecenal and amphotericin B against $A$. fumigatus

\begin{tabular}{lccccl}
\hline \multirow{2}{*}{ Strain } & \multirow{2}{*}{ Agent } & \multicolumn{2}{c}{ MIC values $(\mu \mathrm{g} / \mathrm{ml})$} & \multirow{2}{*}{$\mathrm{FICl}$} & \multirow{2}{*}{ Outcome } \\
& & Alone & Combination & & \\
\hline A. fumigatus & Cis-9-Hexadecanal & 78 & 39 & \multirow{2}{*}{0.74} & Addition \\
ATCC-46645 & Amphotericin B & 6.2 & 1.5 & & \\
\end{tabular}

The results are mean of three different experiments. The results were interpreted as synergy $(\mathrm{FICl} \leq 0.5)$, addition $(0.5<\mathrm{FICl} \leq 1)$, indifferent $(1<\mathrm{FICl} \leq 2)$ or antagonism $(\mathrm{FICl}>2)$.

The conversion of MTT to tetrazolim salt by cellular dehydrogenase was measured which is directly proportional to the number of viable cells present in the biofilm (Manavathu et al., 2014). The MTT assay revealed that there was $80 \%$ eradication of matured biofilm $\left(\mathrm{MBEC}_{80}\right)$ at $0.156 \mathrm{mg} / \mathrm{ml}$ concentration (Fig. 3). There was only $40 \%$ reduction in $\mathrm{AmpB}$ treated biofilm at 4 fold higher concentration than its MIC $(25 \mu \mathrm{g} /$ $\mathrm{ml})$. Class of fatty acid derivative compounds has also been reported to reduce biofilm formation (Rabin et al., 2015). It was evident from in-vitro study that efficacy of AmpB reduced considerably at biofilm stage as are compared to planktonic cells. Gao and Sun, 2015, reported the MIC ranges upto $32 \mu \mathrm{g} / \mathrm{ml}$ for AmpB against $A$. fumigatus biofilm. Combination of the compound together with drugs could result in effective eradication of biofilm (Rabin et al., 2015). The combinatorial application would also reduce the concentrations of both compound and drug.

\section{Scanning electron microscopic study of the biofilm}

SEM analysis was performed to visualize structural organization of the mature biofilm. The micrograph revealed reduced hyphal mat with almost negligible ECM production in $\mathrm{C}-9-\mathrm{H}$ treated biofilm. A. fumigatus biofilm after AmpB treatment was almost similar to that of untreated biofilm as evident by robust mycelia mat formation covered with ECM. In both conditions here were compacted hyphal layering networks, hypha-hypha adhesion at $37^{\circ} \mathrm{C}$ after $24 \mathrm{~h}$ incubation (Fig. 4). Similar results have been reported by Gonzalez-Ramirez et al., 2016. The cell detachment, new conidia, and conidial germination were also observed in this study. Similar results were observed by Melloul et al., 2016.

In-silico screening of compound for therapeutic activity

Computational approach helps to select, rationalize biological activity and predict ADME

Effect of Compound and amphotericin B on A. fumigatus pre-formed biofilm
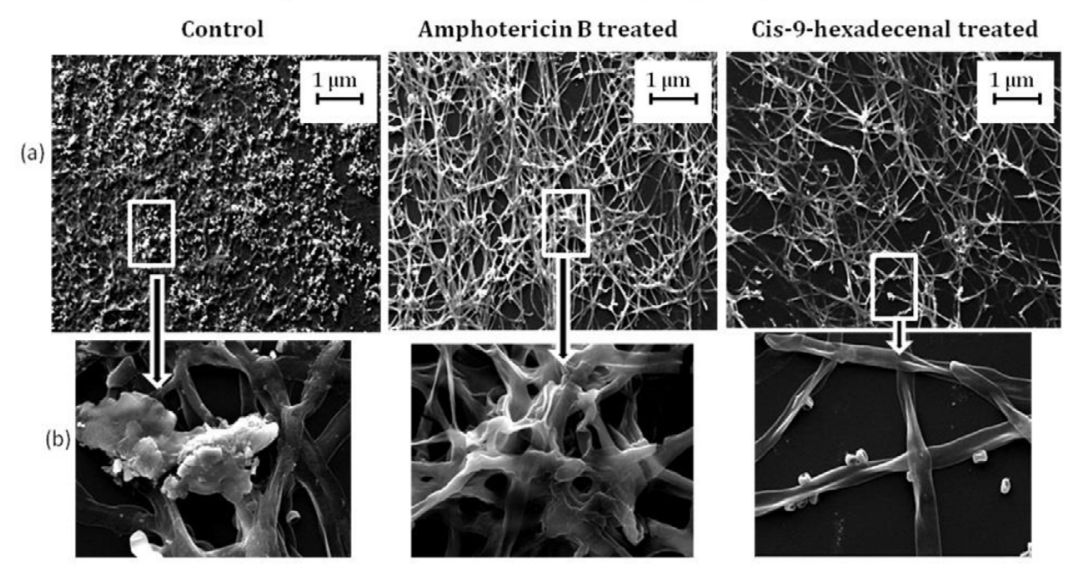

Fig. 4. Scanning electron micrographs illustrating $A$. fumigatus biofilm formation in treated and wild type $A$. fumigatus. The developed biofilm was treated with drug Amphotericin B and cis-9-hexadecenl for $24 \mathrm{~h}$ and then viewed under scanning electron microscope. The magnification of $(a)$ is $500 \mathrm{X}$ and $(\mathrm{b})$ is $5 \mathrm{KX}$. 
properties of natural compounds (Kaserer et al., 2014). Being less expensive technique, these are advantageous over in-vivo studies for preliminary screening. In-silico method provides a high throughput approach avoiding costly late- stage failure in drug development (Guragossian et al., 2016). The ADME/Tox test was conducted to determine the oral bioavailability, health effect, maximum passive adsorption and Central Nervous System (CNS) activity of the ligand. C-9-H has lower molecular weight $(238.41 \mathrm{~g} / \mathrm{mol})$ which would enhance the absorption but due to higher Log $\mathrm{P}$ value (6.29) the compound has lower permeation. This can be enhanced by modifying the structure of the compound. Once drug is administered, ADME/Tox study predicts absorption, distribution, metabolism, excretion and toxic effect of drug. The compound is stable even at $\mathrm{pH}<2$, will undergo passive absorption. The study predicted that after being absorbed in gastrointestinal tract the compound would be delivered to liver where it would metabolize and then reach systemic circulation. In-silico prediction of pharmacokinetic property of $\mathrm{C}-9-\mathrm{H}$ is summarized in Table 1.

The compounds following Lipinski rule are good choice for designing oral drugs (Lipinski et al., 2001). The in-silico analysis of the $\mathrm{C}-9-\mathrm{H}$ revealed that the compound obeys four parameters except the number of rotatable bonds. Rotational bond or hydrogen bond acceptors help to establish quantitative structure activity relationship (QSAR) models to preselect compounds with a higher likelihood of activity on a specific target (Atanasov et al., 2015). It was evident from the results of MolSoft that the compounds have a high log P value. It has been reported that various strategies can be approached for enhancing drug solubility like using novel vehicles, or milling the compound to nano-sized particles; thereby increasing the surface area and facilitating absorption (Westhouse and Car, 2007). Hence, in order to enhance absorption of compound; various modifications can be done in future study. Higher Topological Polar Surface area (TPSA) is better substrate for $p$-glycoprotein which is responsible for drug efflux from cell. Low TPSA makes the substrate better for CNS penetration (Nisha et al., 2016). In present study, TPSA value of $\mathrm{C}-9-\mathrm{H}$ was low (17.07) signifying the fact that the compound may have better CNS penetration.

\section{Cytotoxicity assay against normal lung epithelial cell line L-132}

The cytotoxicity studies using cell-lines are relatively easier to maintain and inexpensive as compared to in-vivo assays (Larsen et al., 2014). The cell line used in this study was L-132 which is normal lung epithelial cell line. Cytotoxicity screening revealed that compound has not inhibited the cell line L-132 proliferation. However, $\mathrm{AmpB}$ reduced the normal cell line viability. Similar results have been reported in earlier studies on various cell lines (Halperin et al., 2016; Zia et al., 2017). The aggregation of $A m p B$ may be the reason of increased cytotoxicity against host cell (Legrand et al., 1992). The compound had no cytotoxic effect on untransformed cell line even at higher concentration of $0.62 \mathrm{mg} / \mathrm{ml}$ whereas the normal antifungal drug AmpB was cytotoxic at $0.025 \mathrm{mg} /$ $\mathrm{ml}$ (Fig. 5).

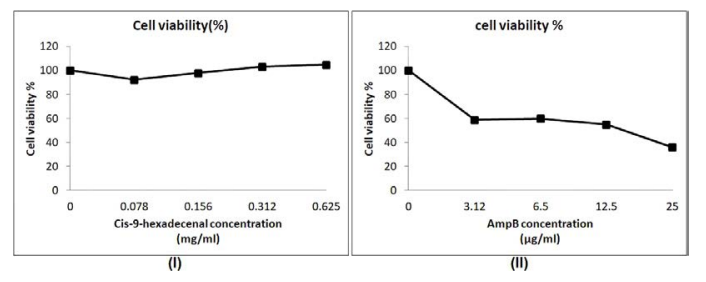

Fig. 5. Cytotoxicity study of cis-9-hexadecenal in comparision to amphotericin B using MTT assay. (I) and (II) are the cell viability assay at various concentrations of cis-9-hexadecenal and amphotericin B respectively.

To understand the effect of $\mathrm{C}-9-\mathrm{H}$ on A. fumigatus at molecular level, future studies will include total proteome and gene expression analysis. This may provide ways of elucidating new therapeutic options for controlling $A$. fumigatus infections.

\section{CONCLUSION}

To the best of our knowledge, present study, for the first time reports the in-vitro antifungal activity of the bioactive compound C-9-H against $A$. fumigatus biofilm. C-9-H possesses great potential in eradicating $A$. fumigatus biofilm and is not cytotoxic to human untransformed cells. Hence, it can be further explored as a lead antifungal molecule against $A$. fumigatus biofilm related infections. 


\section{ACKNOWLEDGEMENTS}

We would like to thank Amity University Uttar Pradesh for providing the infrastructure and facilities for research.

\section{CONFLICT OF INTEREST}

The authors declare that there is no conflict of interest.

\section{AUTHOR'S CONTRIBUTION}

SH carried out the experimental work, data interpretation and prepared manuscript draft. LG, HA and GR helped in part of experiments. MV edited and revised the draft. PV designed the study and supervised the work. SH, LG, HA, GR, MV and $P V$ have read and approved the final manuscript.

\section{FUNDING}

We would like to thank DST-SERB, Govt of India, (EMR/2016/005752) for providing research funding in form of extramural grant.

\section{DATA AVAILABILITY}

All datasets generated during the study are included in the manuscript.

\section{ETHICS STATEMENT}

This article does not contain any studies with human participants or animals performed by any of the authors.

\section{REFERENCES}

1. Nett J.E., Andes D. Fungal Biofilms: In vivo models for discovery of anti-biofilm drugs. Microbiol. Spectr., 2015; 3(3): E30.

2. Lagree K., Desai J.V., Finkel J.S., Lanni F. Microscopy of fungal biofilms. Curr. Opin. Microbiol., 2018; 43: 100107.

3. Sav H., Rafati H., Oz. Y., Dalyan-Cilo B., Ener B., Mohammadi F., et al. Biofilm formation and resistance to fungicides in clinically relevant members of the fungal genus Fusarium. J. Fungi, 2018; 4(1): 16.

4. Martinez L.R., Casadevall A. Cryptococcus neoformans biofilm formation depends on surface support and carbon source and reduces fungal cell susceptibility to heat, cold, and UV light. Appl. Environ. Microbiol., 2007; 73(14): 4592-4601.

5. Fanning S., Mitchell A.P. Fungal biofilms. PLoS Pathog., 2012; 8(4): e1002585.

6. Tseung K.S.Y.N.H., Zhao J. Update on the fungal biofilm drug resistance and its alternative treatment. J. Biosci. Med., 2016; 4(5): 37-47.

7. Liu W., Li L., Sun Y., Chen W., Wan Z., Li R., et al. Interaction of the echinocandin caspofungin with amphotericin B or voriconazole against Aspergillus biofilms in vitro. Antimicrob Agents Chemother, 2012; 56(12): 6414-6416.

8. Boominathan M., Ramamurthy V. Antimicrobial activity of Heliotropium indicum and Coldenia procumbens. J. Ecobiol., 2009; 24(1): 11-15.

9. Veeresham $C$. Natural products derived from plants as a source of drugs. J Adv Pharm Tech Res, 2012; 3(4): 200-201.

10. Ololade Z.S., Fakankun O.A., Alao F.O., Udi O.U. Phytochemical and therapeutic studies of the fruit essential oil of Thuja orientalis from Nigeria. Global J Sci Front Res-B, 2014; 14(7): 96-100.

11. Fang L., Wang X., Guo L., Liu Q. Antioxidant, antimicrobial properties and chemical composition of cumin essential oils extracted by three methods. Open Chem, 2018; 16(1): 291-297.

12. Olasehinde G.I., Mordi R.C., Eluagwulea A.N., Jonathan H.O., Ayo-Ajai J.I., Okedere A.P., et al. Antiplasmodial activity shown by secondary metabolites extracted from the seeds of Pentaclethramacrophylla Benth, 2018; pp. 1-12. In IOP Conf Series: Earth and Environmental Science, Proceedings of the $2 \mathrm{nd}$ International Conference on Science and Sustainable Development.

13. Mujeeb F., Bajpai P., Pathak N. Phytochemical evaluation, antimicrobial activity, and determination of bioactive components from leaves of Aegle marmelos. Biomed. Res. Int., 2014; 2014(Article Id 497606): 1-11.

14. Kumar D., Rajakumar. Gas chromatography-mass spectrometry analysis of bioactive components from the ethanol extract of Avicennia marina leaves. Innovare J Sci, 2016; 4(4): 9-12.

15. Juliet S.Y., Kalimuthu K., Vajjiram C., Ranjitha V. Evaluation and comparison of phytochemical, GCMS and FTIR analysis of wild and micropropagated cadaba fruticosa (L.). World J. Pharm. Res., 2018; 7(14): 746760.

16. Huang Q., Chen L., Song H., An F., Teng H., Xu M. Effect of different drying method on volatile flavor compounds of Lactarius deliciosus. J. Food Process Technol, 2016; 7(8): 615-619.

17. Mei H., Yu X.C., Chan K.K. NB1-C16-Insulin: site-specific synthesis, purification and biological activity. Pharm. Res., 1999; 16(11): 1680-1686.

18. Liu X., Han Y., Peng K., Liu Y., Li J., Liu H. Effect of traditional Chinese medicinal herbs on Candida spp. from patients with HIV/AIDS. Adv. Dent. Res., 2011; 23(1): 56-60.

19. Yang W., Liu J., Blazekovic B., Sun Y., Ma S., Ren C., et al. In vitro antibacterial effects of Tanreqing injection combined with vancomycin or linezolid against methicillin-resistant Staphylococcus aureus. BMC Complement Altern. Med., 2018; 18: 169.

20. Das S., Vasudeva N., Sharma S. Chemical composition of ethanol extract of Macrotyloma uniflorum (Lam.) Verdc. using GC-MS spectroscopy. Org Med Chem Lett, 2014; 4: 13-16.

21. Adams R.P. Identification of essential oil components by gas chromatography/mass spectroscopy, 4th Ed. Allured Publication Corporation, Carol Stream, USA, 2007. 
22. Szumilak M., Galdyszynska M., Dominska K., Bak-Sypien II., Merecz-Sadowska A., Stanczak A., et al. Synthesis, biological activity and preliminary in silico ADMET screening of polyamine conjugates with bicyclic systems. Molecules, 2017; 22(5): 794-816.

23. Clinical and Laboratory Standards Institute (CLSI). 2008. Reference method for broth dilution testing of filamentous fungi, Approved standard- 2nd Ed. CLSI document M38-A2, Wayne, Pennsylvannia, USA.

24. Fratini F., Mancini S., Turchi B., Friscia E., Pistelli L., Giusti G., et al. A novel interpretation of the fractional inhibitory concentration index: The case Origanum vulgare L. and Leptospermum scoparium J. R. et G. Forst essential oils against Staphylococcus aureus strains. Microbiol. Res., 2017; 195: 11-17.

25. Manavathu E.K., Vager D.L., Vazquez J.A. Development and antimicrobial susceptibility studies of in vitro monomicrobial and polymicrobial biofilm models with Aspergillus fumigatus and Pseudomonas aeruginosa. BMC Microbiol, 2014; 14: 53-68.

26. Gonzalez-Ramirez A.I., Ramirez-Granillo A., MedinaCanales M.G., Rodriguez-Tovar A.V., Martinez-Rivera M.A. Analysis and description of the stages of Aspergillus fumigatus biofilm formation using scanning electron microscopy. BMC Microbiol., 2016; 16(1): 243-256.

27. Tambuna U.S.F., Parikesit A.A., Adam V.C., Nasution M.A.F., Puspitasari R.D., Kerami D. Virtual screening of commercial cyclic peptides as $\beta$-OG pocket binder inhibitor in dengue virus serotype 2. Int. J. GEOMATE, 2017; 13(37): 60-68.

28. Lalitha P., Sivakamasundari S. Calculation of molecular lipophilicity and drug likeness for few heterocycles. Orient. J. Chem., 2010; 26(1: 135-141.

29. Lipinski C.A., Lombardo F., Dominy B.W., Feeney P.J. Experimental and computational approaches to estimate solubility and permeability in drug discovery and development settings. Adv. Drug Deliv. Rev., 2001; 46(1-3): 3-26.

30. Yusof I., Shah F., Hashimoto T., Segall M.D., Greene N. Finding the rules for successful drug optimization. Drug Discov. Today, 2014; 19(5): 680-687.

31. Venkatraman M., Anto R.J., Nair A., Verghese M. Karunagaran D. Biological and chemical inhibitors of NF-kB sensitize SiHa cells to cisplatin-induced apoptosis. Mol. Carcinog., 2005; 44(1): 51-59.

32. Jadeja R.N., Vyas K.M., Upadhyay K.K., Devkar R.V. In vitro apoptosis-inducing effect and gene expression profiles of mixed ligand $\mathrm{Cu}$ (II) complexes derived from 4-acyl pyrazolones on human lung cancer cells. RSC Advances, 2017; 7: 17107-17116.

33. Adewole E., Ajiboye B.O., Idris O.O., Ojo O.A., Onikan A., Ogunmodede O.T., et al. Phytochemical, Antimicrobial and Gc-Ms of African Nutmeg (Monodora Myristica). Int. J. Pharm. Sci. Invent, 2013; 2(5): 25-32.

34. Kubo A., Lunde C.S., Kubo I. Antimicrobial activity of the olive oil flavour compounds. J Agri Food Chem, 1995; 43(6): 1629-1633.

35. Bisignano G., Lagana M.G., Trombetta D., Arena S., Nostro A., Uccella N., et al. In vitro antibacterial activity of some aliphatic aldehydes from Olea europaea $\mathrm{L}$. FEMS Microbiol. Lett., 2001; 198(1): 9-13.
36. Trombetta D., Saija A., Bisignano G., Arena S., Caruso S., Mazzanti G., et al. Study on the mechanisms of the antibacterial action, of some plant $\alpha, \beta$-unsaturated aldehydes. Lett App Microbiol, 2002; 35(4): 285-290.

37. Rabin N., Zheng Y., Temeng C.O., Du Y., Bonsu E., Sintim H.O. Agents that inhibit bacterial biofilm formation. Future Med Chem, 2015; 7(5): 647-671.

38. Gao L., Sun Y. In vitro interactions of antifungal agents and tacrolimus against Aspergillus biofilms. Antimicrob. Agents Chemother., 2015; 59(11): 70977099.

39. Melloul E., Luiggi S., Anais L., Arne P., Costa J.M., Fihman V, et al. Characteristics of Aspergillus fumigatus in association with Stenotrophomonas maltophilia in an in vitro model of mixed biofilm. PLoS One, 2016; 11(11): e0166325.

40. Kaserer T., Temml V., Schuster D. Poly-pharmacology and adverse bioactivity profiles predict potential toxicity and drug-related ADRs, 2014, pp. 23-45. In Predictive ADMET: Integrative Approaches in Drug Discovery and Development, Wang J, Urban L (eds.), John Wiley \& Sons, Hoboken, New Jersey.

41. Guragossian N., Gozzi G.J., Fouillet B., Gustin M.P., Terreux R., Bouaziz Z., et al. Candidate molecule selection based on in Silico predicted ADMET properties of 12 indenoindole derivatives. Chem Inform, 2016; 2(2): 6-15.

42. Atanasov A.G., Waltenberger B., Pferschy-Wenzig E.M., Linder T., Wawrosch C., Uhrin P., et al. Discovery and resupply of pharmacologically active plant-derived natural products: A review. Biotechnol Adv, 2015; 33(8): 1582-1614

43. Westhouse R.A., Car B.D. Concepts in pharmacology and toxicology. In Cancer Immunotherapy: Immune Suppression and Tumor Growth, Prendergast GC, Jaffee EM (eds.), Elsevier, USA, 2007; 149-166, Chapter 9 ,

44. Nisha C.M., Kumar A., Nair P., Gupta N., Silakari C., Tripathi T., et al. Molecular docking and in silico ADMET study reveals acylguanidine $7 a$ as a potential inhibitor of $\beta$-secretase. Adv Bioinfo, 2016; 2016(Article ID 9258578): 6.

45. Larsen M.J., Larsen S.D., Fribley A., Grembecka J., Homan K., Mapp A., et al. The role of HTS in drug discovery at the University of Michigan. Comb Chem High Throughput Screen, 2014; 17(3): 210-230.

46. Halperin A., Shadkchan Y., Pisarevsky E., Szpilman A.M., Sandovsky H., Osherov N., et al. Novel water-soluble amphotericin B-PEG conjugates with low toxicity and potent in vivo efficacy. J Med Chem, 2016; 59(3): 11971206.

47. Zia Q., Mohammad O., Rauf M.A., Khan W., Zubair S. Biomimetically engineered amphotericin B nanoaggregates circumvent toxicity constraints and treat systemic fungal infection in experimental animals. Sci rep, $2017 ;$ 7(1): 11873.

48. Legrand P., Romero E.A., Cohen B.E., Bolard J. Effects of aggregation and solvent on the toxicity of amphotericin B to human erythrocytes. Antimicrob. Agents Chemother., 1992; 36(11): 2518-2522. 